\title{
New algorithm and results for the three-dimensional random field Ising Model
}

\author{
J. Machta \\ Department of Physics and Astronomy, University of Massachusetts, Amherst, MA 01003-3720 \\ M. E. J. Newman \\ Santa Fe Institute, 1399 Hyde Park Road, Santa Fe, NM 87501 \\ L. B. Chayes \\ Department of Mathematics, University of California, Los Angeles, CA 90095-1555
}

(October 27, 2018)

\begin{abstract}
The random field Ising model with Gaussian disorder is studied using a new Monte Carlo algorithm. The algorithm combines the advantanges of the replica exchange method and the two-replica cluster method and is much more efficient than the Metropolis algorithm for some disorder realizations. Three-dimensional sytems of size $24^{3}$ are studied. Each realization of disorder is simulated at a value of temperature and uniform field that is adjusted to the phase transition region for that disorder realization. Energy and magnetization distributions show large variations from one realization of disorder to another. For some realizations of disorder there are three well separated peaks in the magnetization distribution and two well separated peaks in the energy distribution suggesting a first-order transition.
\end{abstract}

Typeset using REVTEX 


\section{INTRODUCTION}

Despite twenty five years of experimental and theoretical effort, phase transitions in systems with quenched random fields are still poorly understood. The simplest theoretical model is the random field Ising model (RFIM). The RFIM phase transition is believed to be in the same universality class as the phase transitions in diluted antiferromagnets in a uniform field and fluids in porous media. The three dimensional RFIM is known [1 3] to have an ordered phase at sufficiently low temperature and for weak random fields. As the temperature or the strength of the randomness is increased, there is a transition to a disordered phase. The nature of this transition is not well understood.

In this paper we describe a new replica exchange algorithm for simulating the RFIM and present numerical results for systems of $L \times L \times L$ spins with $L$ up to 24 . We show that the qualitative features of the transition differ strongly from realization to realization of disorder. Our results can be interpreted as suggesting that the RFIM transition is first-order or that a modified version of the droplet picture holds.

The RFIM is described by the energy

$$
-\mathcal{H} / k_{B} T=\beta \sum_{\langle i j\rangle} S_{i} S_{j}+\Delta \sum_{i} h_{i} S_{i}+H \sum_{i} S_{i}
$$

where the spin variables $S_{i}= \pm 1$ reside on a lattice, $\beta=1 / k_{B} T$ is the inverse temperature, the first sum is over nearest neighbor pairs on the lattice, $H$ is an external field and $\Delta$ is the strength of the disorder. The random fields are independent random variables chosen from a distribution with mean zero and variance one. In this study, the random fields are Gaussian and the lattice is simple cubic.

Currently, it is not known whether the phase transition for the 3D RFIM is first-order or continuous. Experiments on magnetic systems have been plagued by problems of poor equilibration and have yielded confusing results, but it appears that there is no latent heat at the transition and this has usually been interpreted as evidence for a continuous transition. Theoretical analyses [- 4 have also favored a continuous transition, although in many cases this is an assumption rather than a conclusion, and some recent work [5] suggests a fluctuation driven first-order transition. The standard picture is that of a continuous transition controlled by a zero temperature fixed point. The scaling theory of this transition [6-8] has three independent critical exponents and modified hyperscaling relations. Because of the fixed point governing the transition has strong disorder, controlled renormalization group calculations have not been possible. Migdal-Kadanoff renormalization group calculations indicate a continuous transition [9,10] but also mistakenly predict that the $q$-state Potts transition is continuous for all $q$ when, for 3D it is known to be first-order for $q \geq 3$. Series analyses initially supported a first-order transition [1] but more recently point to a continuous transition, at least for weak disorder [12]. Alternatively, it may be that the transition is continuous for weak disorder and then becomes first-order for strong disorder with a tricritical point separating the critical line from the first-order line.

Recent Monte Carlo simulations [13 15] have also been interpreted as showing a continuous transitions but with a jump in the magnetization. These simulations have been limited to system size $16^{3}$. The jump in the magnetization can be interpreted as a very small value of the magnetization exponent but might also signal a first-order transition. Simulations of 
systems up to $64^{3}$ [16 were interpreted as indicating a first-order transition but were clearly not equilibrated in the transition region. A number of numerical experiments have also been carried out on the RFIM at zero temperature using polynomial time ground state algorithms. Originally these supported a continuous transition with a small magnetization exponent 17] but more recent studies on larger systems [18] show a jump in the magnetization and are thus suggestive of a first-order transition.

The numerical results presented in this paper differ from those of previous studies in two significant respects. First we use an efficient algorithm that permits us to reach equilibrium for larger systems than those of past studies. Second, we fine-tune both the temperature and the external field for each realization of disorder to be as close as possible to the transition for that realization.

To understand the motivation for this fine-tuning, let us suppose for the moment that the transition is first-order. If this is the case, then for periodic or helical boundary conditions we expect that there will be three phases in coexistence at the transition point. We call the coexisting phases,+- and 0 . The ordered phases, + and - , have long range order and finite magnetization. The disordered, 0 phase has no long range order, no magnetization and is characterized by spins that are predominately aligned with their local fields. The bond energy, defined by the first term on the RHS of Eq. (1.1), is greater in the 0 phase than the ordered phases. The expected phase diagram in the $T-H$ plane in the vicinity of the point of three phase coexistence is shown in Fig. 1. For $T<T_{c}$ and $H=0$ there is phase coexistence of the + and - phases that ends at the thermal first-order transition at $T=T_{c}$ and $H=0$ (the black dot in the figure). Since the 0 and $+(-)$ phases differ in both energy and magnetization, the disordered phase can be maintained in coexistence with the $+(-)$ phase by increasing the temperature and increasing (decreasing) the field. The two order-disorder lines corresponding to $0+$ and $0-$ coexistence form the arms of the "Y" in the figure.

Since the disorder is independent and homogenous the free energy is self-averaging, hence, in the thermodynamic limit the transition occurs at a definite point $\left(T_{c}, H=0\right)$ for almost all realizations of the random fields. However, for finite systems, the location and qualitative features of the transition will depend on the realization of disorder. In particular, for a system of size $L$, with realization of random field, $\left\{h_{i}\right\}$ and disorder strength $\Delta$ there may be a point where three phases coexist at $\left(T_{c}\left(\Delta ;\left\{h_{i}\right\}\right), H_{c}\left(\Delta ;\left\{h_{i}\right\}\right)\right)$. A first guess, based on the net field due to the random fields, is that $T_{c}\left(\Delta ;\left\{h_{i}\right\}\right)$ and $\left.H_{c}\left(\Delta ;\left\{h_{i}\right\}\right)\right)$ should be displaced from the average value $\tilde{T}_{c}(L)$ and $H=0$ by an amount of order $\Delta L^{d / 2}$.

How accurately must $H$ and $T$ be fine-tuned to see three phase coexistence if it exists? Suppose first that we want the + and - phases to coexist and that the magnetizations of these phases at the transition differs by $2 m$. If the external field deviates from the correct value by $\delta H$, the free energy difference between the + and - phase is $2 m \delta H L^{d}$. For both phases to have a significant probability the free energy difference must not greatly exceed $k T$. Thus $H$ must be set to $H_{c}\left(\Delta ;\left\{h_{i}\right\}\right)$ to within an accuracy of $k T / 2 m L^{d}$ to have both phases represented. $H_{c}\left(\Delta ;\left\{h_{i}\right\}\right)$ will itself fluctuate from sample to sample as $\Delta / L^{d / 2}$. Similarly, if the entropy difference per spin between the the ordered and disordered phases is $s$, then $T$ must be fine-tuned to within $k T / s L^{d}$ to allow to these phases to coexist. Presumably sample to sample fluctuations in $T_{c}\left(\Delta ;\left\{h_{i}\right\}\right)$ also scale as $1 / L^{d / 2}$. Thus if a single value of $H=0$ and $T=\tilde{T}_{c}(L)$ is chosen for all realizations of the random field, one will almost never 
see more than one phase at a time.

\section{NUMERICAL METHODS}

We use an algorithm that combines the replica exchange method, first introduced by Swendsen and Wang [19] and the two-replica cluster method of Redner, Machta and Chayes 20,21]. Our method is also closely related to simulated and parallel tempering [22]. The idea of this approach is to simultaneously simulate $K$ replicas of the system. All replicas have the same normalized random field $\left\{h_{i}\right\}$ but each replica has different values of the other parameters. Replica $k(k=0, \ldots, K-1)$ has inverse temperature $\beta_{k}$, strength of randomness $\Delta_{k}$ and external field $H_{k}$. The replicas form a sequence so that neighboring replicas in the sequence are nearby in the $(\beta, \Delta, H)$ parameter space. Neighboring replicas exchange magnetization with one another according to a procedure described below. One end of the sequence of replicas is at a value of $\beta, \Delta$ and $H$ that can be efficiently simulated using a known method while the other end of the sequence is at a value of the parameters that we would like to study. In our case, the replicas lie along the RFIM phase transition line starting from the pure Ising values $\beta_{0} \approx .22615$ and $\Delta_{0}=H_{0}=0$ as shown in Fig. 2. The replicas are equally spaced in $\Delta$. The pure Ising replica is simulated using the Wolff single cluster algorithm [23,24]. (We have also experimented with replicas lying along a line of constant $\beta H$ starting at the RFIM phase boundary, extending into the paramagnetic phase, and ending at a temperature high enough that the model can be efficiently simulated using the ordinary single-spin-flip Metropolis algorithm. This approach, however, is found to be less efficient than the one described above.)

Magnetization is exchanged between neighboring replicas using a generalization of the two-replica cluster method. Suppose we have two replicas at $(\beta, \Delta, H)$ and $\left(\beta^{\prime}, \Delta^{\prime}, H^{\prime}\right)$ with spin configurations $\left\{S_{i}\right\}$ and $\left\{S_{i}^{\prime}\right\}$, respectively. A site $j$ is considered active for this pair of replicas if $S_{j} \neq S_{j}^{\prime}$. A bond $i j$ between neighboring sites $i$ and $j$ is satisfied if $S_{i}=S_{j}$ and $S_{i}^{\prime}=S_{j}^{\prime}$. A cluster of active sites is formed starting from a randomly chosen active site. New active sites are added to the cluster by occupying satisfied bonds on the perimeter of the cluster with probability $p\left(\beta, \beta^{\prime}\right)$ where

$$
p\left(\beta, \beta^{\prime}\right)=1-e^{-2\left(\beta+\beta^{\prime}\right)} .
$$

If a bond connecting a site to a cluster is occupied, the site is added to the cluster and the set of bonds on the perimeter is updated. In this way, the cluster grows until no further sites are added. The procedure is very similar to the way clusters are grown in the Wolff single cluster algorithm.

Once a cluster is identified it is flipped with a probability that depends on the change in boundary and field energy so as to satisfy detailed balance. Flipping a cluster means changing the sign of all the spins in the cluster or, equivalently, exchanging the values of the spins in the cluster between the two replicas. The probability to flip a cluster $\mathcal{C}$ with $|\mathcal{C}|$ sites depends on the quantity $\Sigma$

$$
\Sigma=-\left[\left(\Delta-\Delta^{\prime}\right) h_{\mathcal{C}}+\left(H-H^{\prime}\right)|\mathcal{C}|+\left(\beta-\beta^{\prime}\right)\left(N_{++}-N_{--}\right)\right] S_{\mathcal{C}}
$$

where $N_{++}$and $N_{--}$are, respectively, the number of ++ and -- sites that are nearest neighbors of the cluster, $h_{\mathcal{C}}$ is the net random field acting on the cluster, 


$$
h_{\mathcal{C}}=\sum_{i \in \mathcal{C}} h_{i},
$$

and $S_{\mathcal{C}}$ is the spin value of the cluster in the unprimed replica. If $\Sigma \leq 0$ then the cluster is flipped, otherwise it is flipped with probability $e^{-\Sigma}$.

It is straightforward but tedious to show that the choice of $p\left(\beta, \beta^{\prime}\right)$ (Eq. 2.1) together with the flipping probability defined by $\Sigma$ is precisely what is needed to ensure detailed balance. The motivation for the choice of $p\left(\beta, \beta^{\prime}\right)$ is most easily understand by considering the limit where two replicas are at the same values of $\beta, \Delta$ and $H$. In that case $p(\beta, \beta)=1-e^{-4 \beta}$ and $\Sigma=0$. Since $\Sigma=0$, clusters are always flipped just as is the case for the Wolff single cluster algorithm. Furthermore, we have shown in Ref. [20,21] that the active clusters percolate at the RFIM phase transition. If the transition is continuous clusters of all sizes are flipped. If the transition is first-order, there will be two distinct kinds of clusters; some clusters will be extensive and change the phase of the system while other clusters will have sizes less than or equal to the correlation length. In either case, the clusters identified by the two-replica procedure correspond to the fluctuations that actually occur in the system at the phase transition and permits large changes in the spin configuration in a single Monte Carlo sweep. When the two replicas do not have equal values of the parameters, then the clusters do not flip freely but if the replicas are close together in the parameter space, the acceptance fraction for flipping clusters will remain high.

Our method and the original replica exchange method [19] on which it is based is similar to parallel tempering [22]. In all these methods, groups of spins are exchanged between neighboring replicas along a sequence. In parallel tempering the whole spin configuration is exchanged and the Boltzmann factor controlling the acceptance of the move depends on the energy difference between the replicas. In our algorithm, only some of the spins are exchanged and for a given distance in the parameter space between the replicas the acceptance fraction is larger than for parallel tempering. The consequence is that fewer, less closely spaced replicas are needed for the replica exchange method.

In order to find the phase transition temperature and external field $\left(\beta_{c}\left(\Delta ;\left\{h_{i}\right\}\right)\right.$, $\left.H_{c}\left(\Delta ;\left\{h_{i}\right\}\right)\right)$ for a given realization and strength of disorder we use a feedback mechanism. Starting from an initial value of $\beta$ and $H$ we monitor the magnetization of the system after each Monte Carlo sweep. If the absolute value of the magnetization is less than a lower cut-off $M_{l c}$ the system is interpreted to be in a high temperature phase and the inverse temperature is increased by a small amount $\epsilon_{\beta}$. If the absolute value of the magnetization is greater than an upper cut-off $M_{u c}$ the system is interpreted to be in one of the ordered phases and $\beta$ is decreased by $\epsilon_{\beta}$. In this case, the external field is also adjusted by an amount $\epsilon_{H}$ if the magnetization is positive and by $-\epsilon_{H}$ if the magnetization is negative. The average value of $\beta$ and $H$ is computed for the period when the feedback procedure is on and is taken to be $\left(\beta_{c}\left(\Delta ;\left\{h_{i}\right\}\right), H_{c}\left(\Delta ;\left\{h_{i}\right\}\right)\right)$. The feedback procedure is then turned off and these values are used for a long equilibrium simulation.

Most of our simulations were for $24^{3}$ systems. Except as otherwise noted, the simulations used $K=16$ replicas equally spaced in $\Delta$ with the most disordered replica having $\Delta=.35$. Initially the replicas lie on an elliptical curve in the temperature-disorder plane that starts at the pure Ising transition $(\beta=0.22165)$ and ends at the zero temperature transition $(\Delta / \beta=2.35)$ as shown in Fig. 2. It was shown in Ref. 15 that this curve is a good estimate for the phase boundary. The feedback parameters were $M_{l c}=2000, M_{u c}=4000$, 
$\epsilon_{\beta}=10^{-4}$ and $\epsilon_{H}=10^{-5}$. The feedback mechanism was run for $2 \times 10^{5}$ Monte Carlo sweeps and then, with the temperature and external field determined by the feedback procedure, data were collected for $8 \times 10^{5}$ Monte Carlo sweeps. Each Monte Carlo sweep is defined as 500 cluster steps; since the average cluster size was found to be roughly 1000 , a single Monte Carlo sweep corresponds to attempting to flip every spin in every replica about once. For each cluster step a random integer $k$ was chosen between 0 and $K$. If $k=0$, a Wolff move was performed on the pure replica, if $1<k<K$, a two replica cluster move was carried out between replica $k$ and replica $k-1$. If $k=K$, a two replica cluster move was carried out between replicas $K-1$ and $K-2$. The procedure described above, totaling $10^{6}$ sweeps, was performed for 22 realizations of disorder, a number chosen in advance of the experiment, labeled 10 through 31 by the seed of the random number generator that produced $\left\{h_{i}\right\}$. Each realization required about six days on a $450 \mathrm{MHz}$ Pentium III machine. A number of additional simulations were carried out for specific realizations of disorder (not all in the range 10 through 31) using parameters that differed in some way from the above. In particular, we did a careful study of realization 1 that is described below.

\section{RESULTS}

\section{A. Energy and magnetization histograms}

The main lesson of our work is that each realization of disorder has its own character. This is best seen by examining the probability distributions of magnetization and energy

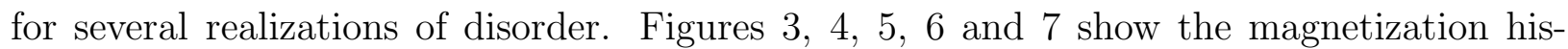
togram for realizations $20,21,25,31$ and 14 , respectively. The magnetization histogram for realization 20 , characterized by two broad maxima roughly symmetrical about the origin, is typical of the majority of realizations. Apart from the asymmetry in the two peaks, realization 20 and those like it are similar to the pure Ising model whose magnetization histogram is shown in Fig. 8 at the infinite system size critical temperature, $\beta_{c}(0)=.22165$. The magnetization histogram for realization 21, Fig. 团also displays two peaks but now one peak is much sharper than the other and quite asymmetric about the origin. The magnetization histogram for realizations 25, Fig. 5 and 31, Fig. 6 are qualitatively different, displaying three distinct and well separated peaks. It should be noted that the feedback procedure was initially unsuccessful for these realizations and had to be run again with $M_{l c}=M_{u c}=5000$ to find all three peaks. Failure of the feedback scheme was evidenced by the presence of only one narrow peak in the magnetization histogram. In the case of realization 31, the feedback procedure with the revised parameters failed on the most disordered replica so Fig. 6 shows the second to last replica in the sequence at $\Delta=.3267$. For both realization 25 and 31 , the original run showed three peaked structures at weaker disorder giving a strong hint that the same qualitative feature would be found at stronger disorder. Finally, realization 14, Fig. 0 was unique, displaying a five peaked structure. Out of a total of 22 magnetization histograms, 19 show two peaks, 2 three peaks and 1 five peaks. It is possible but unlikely that some of the two peak realizations would display more peaks with different fine-tuning. For example, we used other alternate values of the fine-tuning parameters to search for an additional positive peak for realization 21 but none was found. 
The bond energy distribution displayed two possible qualitative behaviors. Figures 9 , 10, 11, 12 and 13 show the bond energy histogram for realizations 20, 21, 25, 31 and 14, respectively. For comparison, Fig. 14 shows the energy distribution for the pure Ising model at the infinite system size critical temperature. For realization 20 and those like it, the bond energy distribution has a single peak and is qualitatively like that of the pure Ising model at criticality, except that the peak is shifted to significantly lower energies. For realization 21, which has two well separated magnetization peaks, there are also two well separated peaks in the bond energy distribution. Similarly, for realizations 25 and 31 there are two peaks in the bond energy distribution. In a first-order interpretation of these realizations the + and - phases have the lower energy and the 0 phase the higher. On the other hand, for realization 14, which has five magnetization peaks, there is a single broad maximum in the bond energy histogram with a significant, low energy shoulder. Altogether, 5 out of 22 realizations show two well defined peaks in the bond energy histogram while the remaining realizations show a single peak though sometimes with a significant low energy shoulder.

The joint magnetization/bond-energy distribution for realization 25 is shown in Fig. 15. For comparison, the joint distribution for the pure Ising model at the infinite system critical temperature is shown in Fig. 16. The three lobes in the distribution correspond to the three peaks in the magnetization histogram, Fig. 5. It is tempting to interpret realizations 25 and 31 in the language of first-order transitions and declare the low energy side lobes as ordered phases and the central lobe as a disordered phase.

The importance of fine-tuning the magnetization and temperature for each realization of disorder is illustrated in Figs. 17, 18, 19 and 20. The magnetization histogram for realization 1 at the fine-tuned parameter values, $\beta_{c}=0.268385$ and $H_{c}=0.00127049$ is shown in Fig. 17. Note, that this value of $H_{c}$ is not the same as the value that would be obtained by summing the local fields. For realization one, that value is almost $50 \%$ larger, $-\Delta \sum_{i} h_{i} / N=0.00178774$. Figure 18 shows realization 1 with same external field but with the temperature raised by $5 \%$ leaving only the disordered phases. Figure 19 shows realization 1 with the same external field but the temperature decreased by $5 \%$. Although, the external field is too negative here, it is nonetheless clear that the two ordered phases are now dominant over the disordered phase, which has nearly vanished. Finally, if the external field is set to 0 and the feedback temperature is used, only the negative peak survives, as shown in Fig. 20.

We considered several realizations at stronger disorder. Figure 21 shows the magnetization histogram for realization 1 at disorder $\Delta=0.433$ at the value of external field and temperature determined from the fine-tuning procedure. Comparing Fig. 21 to Fig. 17 we see that the three-peaked structure is conserved and the peaks becomes sharper as the strength of the disorder is increased. In this experiment, we used 16 replicas with maximum disorder strength, $\Delta=0.5$ however, the fine-tuning procedure failed for the two most disordered replicas and only yielded the minus phase. In general, the feedback procedure was less stable for stronger disorder. Figure 22 shows realization 14 at disorder $\Delta=0.5$ at the values of the temperature and external field determined from the fine-tuning procedure. A comparison to Fig. 0 shows that increasing the disorder has sharpened the five peaked structure of the magnetization histogram. It would be interesting to carry out a systematic study of stronger disorder to see if there is a trend toward more "first-order" like realization however this will require developing a better fine-tuning mechanism. 
Overall, out of 21 realizations (seeds 10-30) at disorder strength $\Delta=.35$ we found that the average values of the critical parameters is $\left\langle\beta_{c}\right\rangle=0.2663$ and $\left\langle H_{c}\right\rangle=0.0006$ (compared with initial values before the feedback procedure of $\beta=0.2670$ and $H=0$ ) with a standard deviations of $\sigma_{\beta}=0.0037$ and $\sigma_{H}=0.0031$. The standard deviation of $H_{c}$ is consistent with the anticipated value $\sigma_{H} \sim \Delta L^{-d / 2}=0.0030$.

\section{B. Dynamics of the algorithm}

We have studied the dynamics of the replica exchange algorithm and compared it to the Metropolis algorithm. There are two ways of comparing the algorithms. First, time can be measured by Monte Carlo sweeps. This approach favors the replica exchange algorithm since a single Monte Carlo sweep involves flipping spins in many (here 16) replicas and since growing clusters is computationally more intensive than flipping single spins. The second approach is to compare actual running time for the two algorithms on the same computer. This approach ignores the possibility that the one algorithm is better optimized and it is not an appropriate way to measure fundamental quantities such as the dynamic exponent however it does give a practical comparison and is useful for deciding which algorithm to choose for a given system size. For $24^{3}$ systems with 16 replicas with maximum disorder $\Delta=.35$ on a $450 \mathrm{MHz}$ Pentium III machine we find that the replica exchange algorithm runs at about $0.56 \mathrm{sec} / \mathrm{sweep}$ and that our implementation of the Metropolis algorithm runs at about $0.0088 \mathrm{sec} / \mathrm{sweep}$. As is the case for the equilibrium properties of the system, we find great differences in the dynamics depending on the realization of disorder. These differences are much more pronounced for the Metropolis algorithm. We measured the integrated autocorrelation time for the magnetization for realization 20 for both algorithms with the result $\tau^{\text {Metropolis }}=6000$ and $\tau^{\text {replica }}=1100$ when measured in Monte Carlo sweeps. The advantage of the replica exchange algorithm is lost however when these times are converted to running times; $\tau^{\text {Metropolis }}=53 \mathrm{~s}$ and $\tau^{\text {replica }}=616 \mathrm{~s}$. The joint energy $/$ magnetization histogram for realization 20 is smooth and without gaps suggesting that there are no global energy barriers separating regions of phase space. At the other extreme is realization 31 for which the joint energy/magnetization distribution is split into three pieces separated by gaps where the probability is very small. Using the parameters for realization 31 determined by fine-tuning for $\Delta=.3267$, we did a long Metropolis run of 100 million Monte Carlo sweeps starting from random initial spins. The simulation stayed mainly in the "0" state with two excursions to the "+" state. The "-" state was never visited. On the other hand, two Metropolis runs of 20 million sweeps starting from all spins up and all spins down went to the "0" and "-" states, respectively, and stayed there for the entire simulation. Figure 23 shows the magnetization time series from the replica exchange algorithm for realization 31 for 800,000 sweeps. Although it is difficult to estimate the autocorrelation time from this series, it is clear that the simulation samples each of the states many times. The conclusion is that for most realizations of disorder, at size $24^{3}$ there is no great practical advantage to using the replica exchange algorithm but that for cases where well separated regions are present in the joint distribution, only the replica exchange algorithm is able to reach equilibrium within reasonable simulation times. 


\section{DISCUSSION}

One of the chief motivations for this study was to determine the nature of the RFIM phase transition. Unfortunately, our results fail to settle this question. What would we have expected from the different scenarios for the phase transition? If the transition is first-order we should find three phases in coexistence at the transition point. The high temperature phase, would have little magnetization and a large bond energy while the two low temperature phases would have large absolute values of magnetization, one positive and the other negative, and small bond energies. This situation is exactly what is seen in roughly $10 \%$ of realizations. For systems much smaller than $24^{3}$, three phase coexistence is not seen simply because the magnetization distribution is too broad to display three distinct peaks. One hypothesis is that, as system size becomes large, the fraction of systems displaying three phases increases and that, in the thermodynamic limit, the transition at $\Delta=.35$ is first-order. However, the existence of realization 14 with its five peaks is difficult to reconcile with this viewpoint.

If the transition is continuous and follows the droplet model scenario [6,86,25] we would expect two peaks in the magnetization histogram corresponding to two states of a single critical phase. The width of each peak should scale as $L^{-\gamma / \nu}$ and the separation between the peaks should scale as $L^{-\beta / \nu}$. The majority of our disorder realizations display two peaks and an alternative to the first-order transition hypothesis is that, as system size increases, the fraction of realizations with two peaks in the magnetization histogram approaches $100 \%$. Although the original droplet model envisioned two states with roughly the same energy and differing by flipping a critical cluster of spins, it is possible to imagine more complicated droplet pictures where there are sometimes more than two states in the critical phase and therefore more than two peaks in the magnetization histogram. However, our five realizations that display more than one peak in the bond energy histogram are difficult to reconcile with the droplet model since one expects the states, however many there are, to be nearly degenerate in bond energy.

Ultimately, the essential difference between the first-order scenario and the droplet model continuous-transition scenario is whether there are distinct phases at the transition point or whether the peaks in the magnetization histogram are different states that are part of a single critical phase. The comes down to the question of whether the peaks in magnetization histogram move toward the origin as $L \rightarrow \infty$ or remain at finite values. Unfortunately, the consensus is that the magnetization exponent, if it is not actually zero, is very tiny so that the decrease in magnetization with system size would be far too weak to observe in simulations.

\section{CONCLUSIONS}

We have studied the phase transition of the random field Ising model by Monte Carlo simulation of systems of size $24^{3}$ using a new cluster algorithm. We find major qualitative differences between the behavior of different realizations of disorder. Some realizations display a broad two-peaked magnetization histogram consistent with a continuous transition, while a small fraction display a three-peaked structure consistent with a first-order transition.

Our main conclusion is that more work needs to be done to determine the nature of the 
transition. It would be very useful to study larger system sizes and stronger disorder to determine whether there are trends in the qualitative features of the phase transition. Does the fraction of "first-order" systems increase as disorder or system size increases? It will require improvements of the algorithm including the fine-tuning technique or substantially more computer power to resolve these questions.

\section{ACKNOWLEDGEMENTS}

We thank Pozen Wong and Robert Swendsen for useful discussions. This work was supported by NSF grants DMR-9978233 (JM) and DMS-9971016 (LC) and NSA grant MDA904-98-1-0518 (LC). JM thanks the Santa Fe Institute for hospitality during a visit in which some of this work was carried out. 


\section{REFERENCES}

[1] Y. Imry and S. K. Ma. Random-field instability of the ordered state of continuous symmetry. Phys. Rev. Lett., 35:1399, 1975.

[2] J. Z. Imbrie. Lower critical dimension of the random-field Ising model. Phys. Rev. Lett., $53: 1747,1984$.

[3] J. Bricmont and A. Kupiainen. Lower critical dimension for the random-field Ising model. Phys. Rev. Lett., 59:1829, 1987.

[4] T. Nattermann. Theory of the random field Ising model. In A. P. Young, editor, Spin Glasses and Random Fields. World Scientific, Singapore, 1997.

[5] E. Brezin and C. De Dominicis. New phenomena in the random field Ising model. Europhys. Lett., 44:13, 1998.

[6] J. Villain. Equilibrium critical properties of random field systems: New conjectures. J. Physique, 46:1843, 1985.

[7] A. J. Bray and M. A. Moore. Scaling theory of the random-field Ising model. J. Phys. $C, 18: L 927,1985$.

[8] D. S. Fisher. Scaling and critical slowing down in random-field Ising systems. Phys. Rev. Lett., 56:416, 1986.

[9] M. S. Cao and J. Machta. Migdal-Kadanoff study of the random field Ising model. Phys. Rev. B, 48, 1993.

[10] A. Falicov, A. N. Berker, and S. R. McKay. Renormalization-group theory of the random-field Ising model in three dimensions. Phys. Rev. B, 51:8266, 1995.

[11] A. Houghton, A. Khurana, and F. J. Seco. Fluctuation-driven first-order phase transition, below four dimensions, in the random-field Ising model with a gaussian randomfield distribution. Phys. Rev. Lett., 55, 1985.

[12] M. Gofman, J. Adler, A. Aharony, A. B. Harris, and M. Schwartz. Critical behavior of the random field Ising model. Phys. Rev. B, 54:364, 1996.

[13] H. Rieger and A. P. Young. Critical behavior of the three-dimensional random-field Ising model: Two-exponent scaling and discontinuous transition. Phys. Rev. B, 52:6659, 1995.

[14] H. Rieger. Critical behavior of the three dimensional random field Ising model. J. Phys. A: Math. Gen., 26:5279, 1993.

[15] M. E. J. Newman and G. T. Barkema. Monte Carlo study of the random-field Ising model. Phys. Rev. E, 53:393, 1996.

[16] A. P. Young and M. Nauenberg. Quasicritical behavior and first-order transition in the $d=3$ random-field Ising model. Phys. Rev. Lett., 54:2429, 1985.

[17] Ogielski. Integer optimization and zero-temperature fixed point in Ising random-field systems. Phys. Rev. Lett., 57:1251, 1986.

[18] N. Sourlas. Universality in random systems: The case of the 3-d random field Ising model. Comput. Phys. Commun., 122:183, 1999.

[19] R. H. Swendsen and J.-S. Wang. Replica Monte Carlo simulations of spin glasses. Phys. Rev. Lett., 57:2607-2609, 1986.

[20] O. Redner, J. Machta, and L. F. Chayes. Graphical representations and cluster algorithms for critical points with fields. Phys. Rev. E, 58:2749-2752, 1998.

[21] L. Chayes, J. Machta, and O. Redner. Graphical representations for Ising systems in external fields. J. Stat. Phys., 93:17, 1998. 
[22] E. Marinari and G. Parisi. Simulated tempering: A new Monte Carlo scheme. Europhys. Lett., 19:451, 1992.

[23] U. Wolff. Collective Monte Carlo updating for spin systems. Phys. Rev. Lett., 62:361, 1989.

[24] M. E. J. Newman and G. T. Barkema. Monte Carlo Methods in Statistical Physics. Oxford, Oxford, 1999.

[25] K. Eichorn and K. Binder. Monte carlo investigation of the three-dimensional randomfield three-state Potts model. J. Phys. A: Condens. Matter, 8:5209-5227, 1996. 


\section{FIGURES}

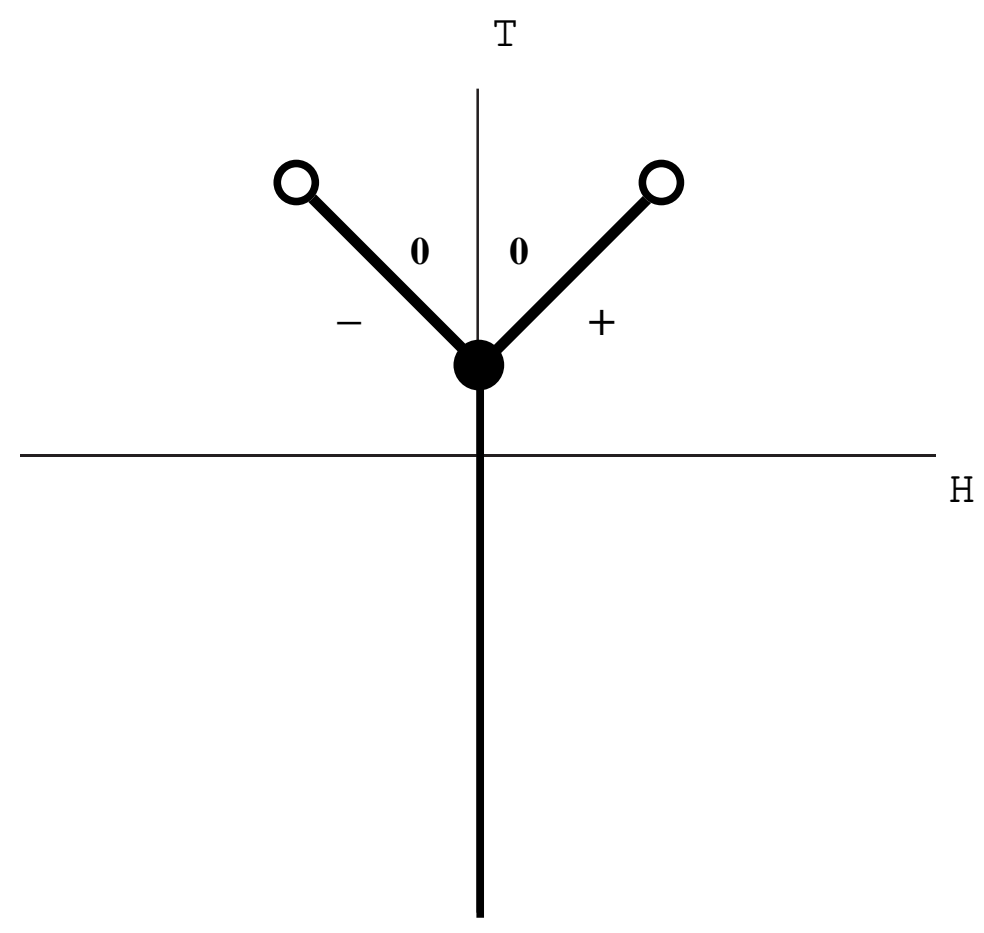

FIG. 1. Proposed phase diagram for the 3D RFIM in the $H-T$ plane at a fixed strength of disorder. The bold lines are first-order lines. The black dot is the thermal first-order transition at $\left(T_{c}, H=0\right)$ where the,+- and 0 phases coexist. The open circles are critical endpoints of the two order-disorder first-order lines.

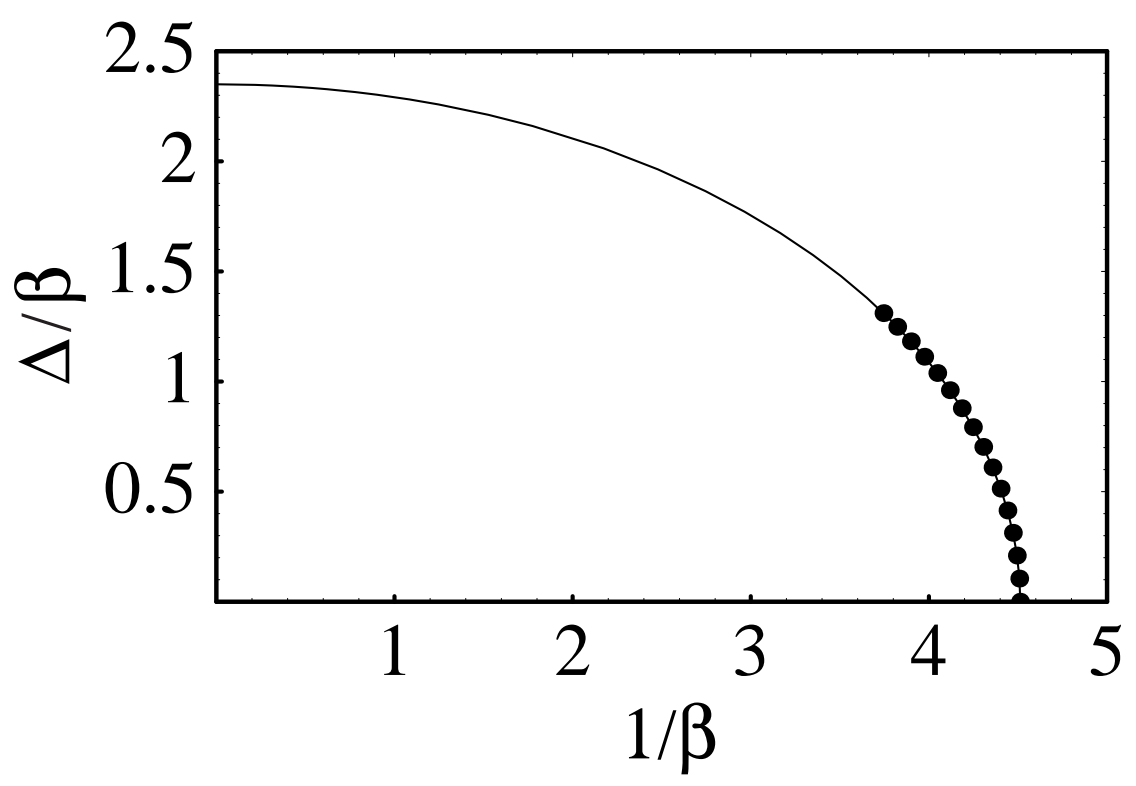


FIG. 2. Approximate phase diagram for the RFIM and replicas for the replica exchange method. The phase boundary in the $1 / \beta-\Delta / \beta$ plane is taken to be an elliptical curve starting at the pure Ising critical point $(1 / \beta=4.512, \Delta / \beta=0)$ and ending at the zero temperature transition $(1 / \beta=0, \Delta / \beta=2.35)$. The initial conditions for the 16 replicas lie on this curve and are evenly space in $\Delta$. During the feedback process, each replica is shifted by a small amount in $\beta$ and $H$.

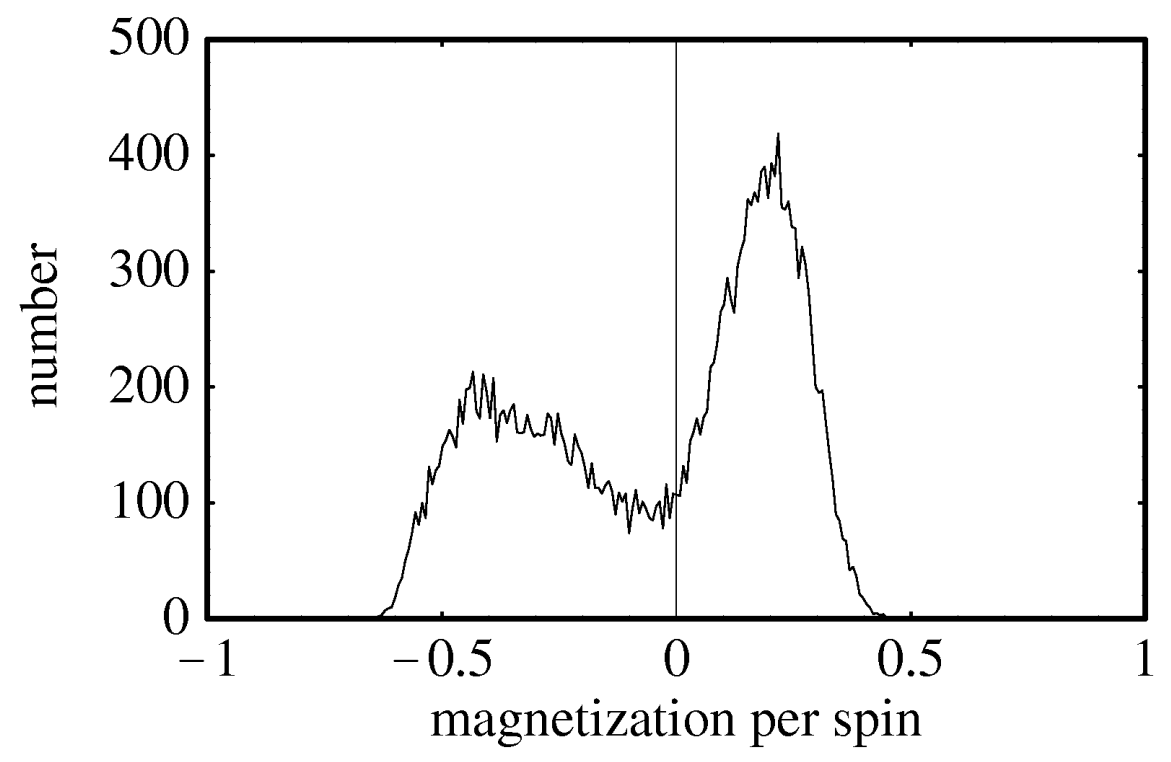

FIG. 3. Magnetization histogram for realization 20 at $\Delta=0.35$.

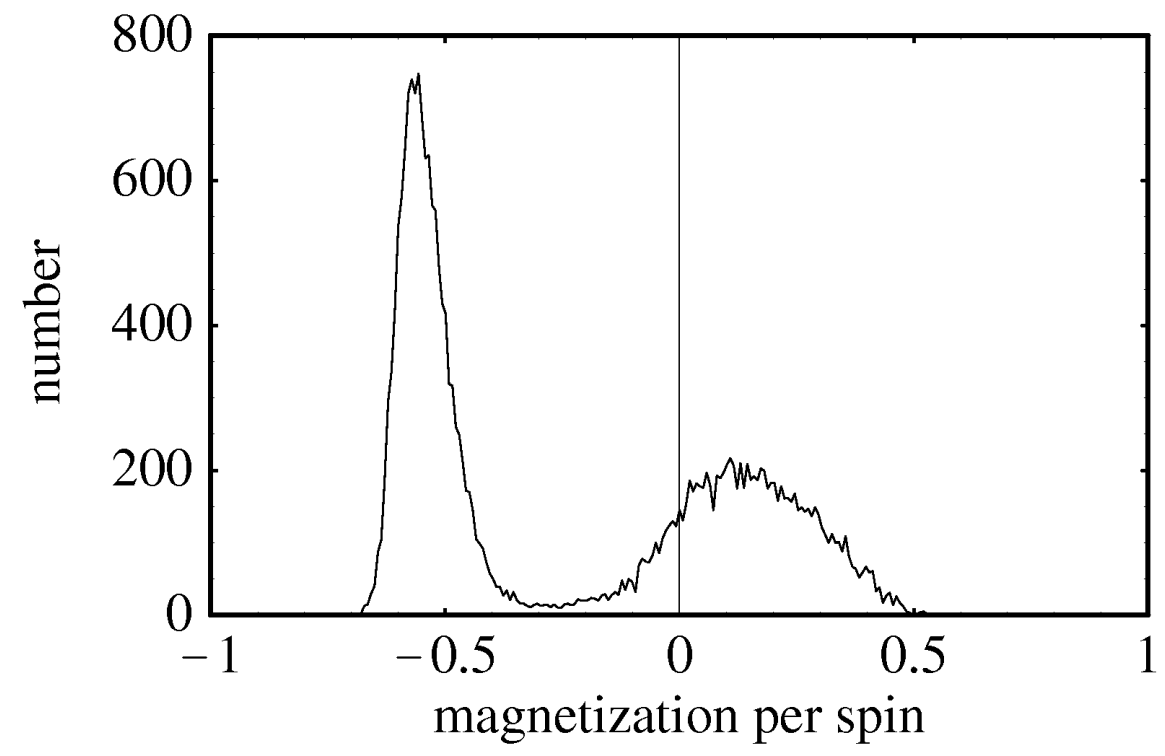

FIG. 4. Magnetization histogram for realization 21 at $\Delta=0.35$. 


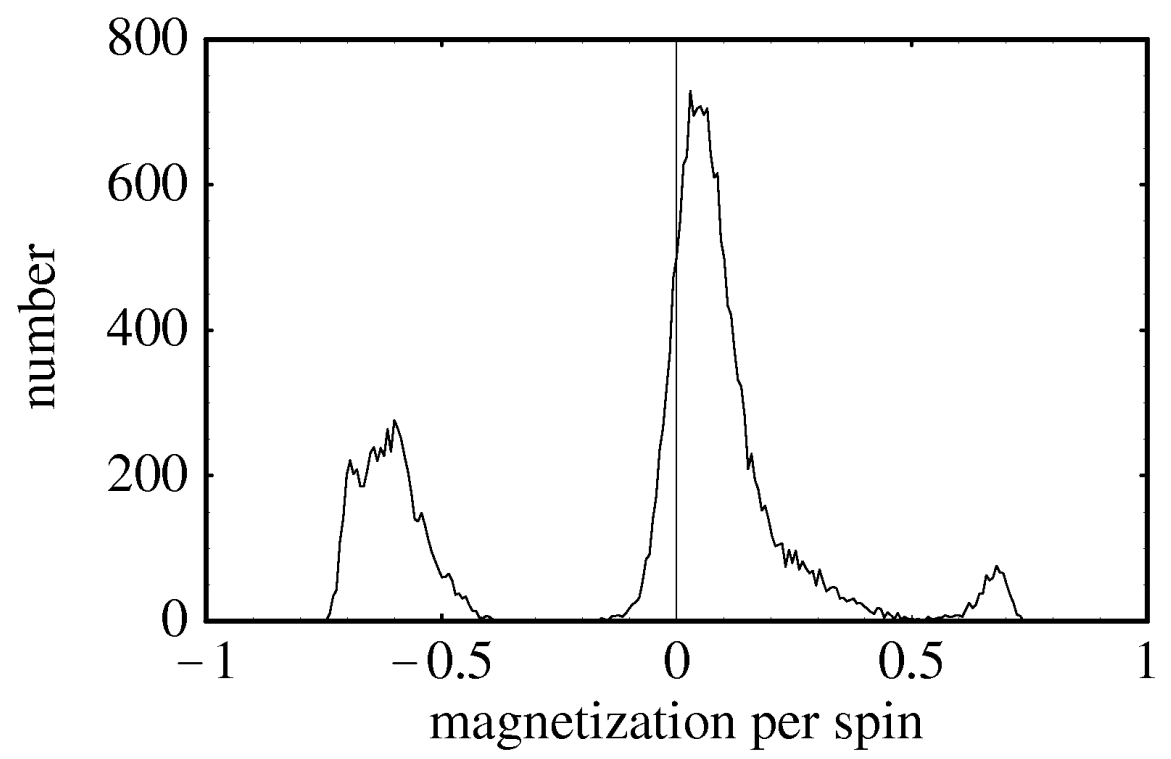

FIG. 5. Magnetization histogram for realization 25 and $\Delta=0.35$.

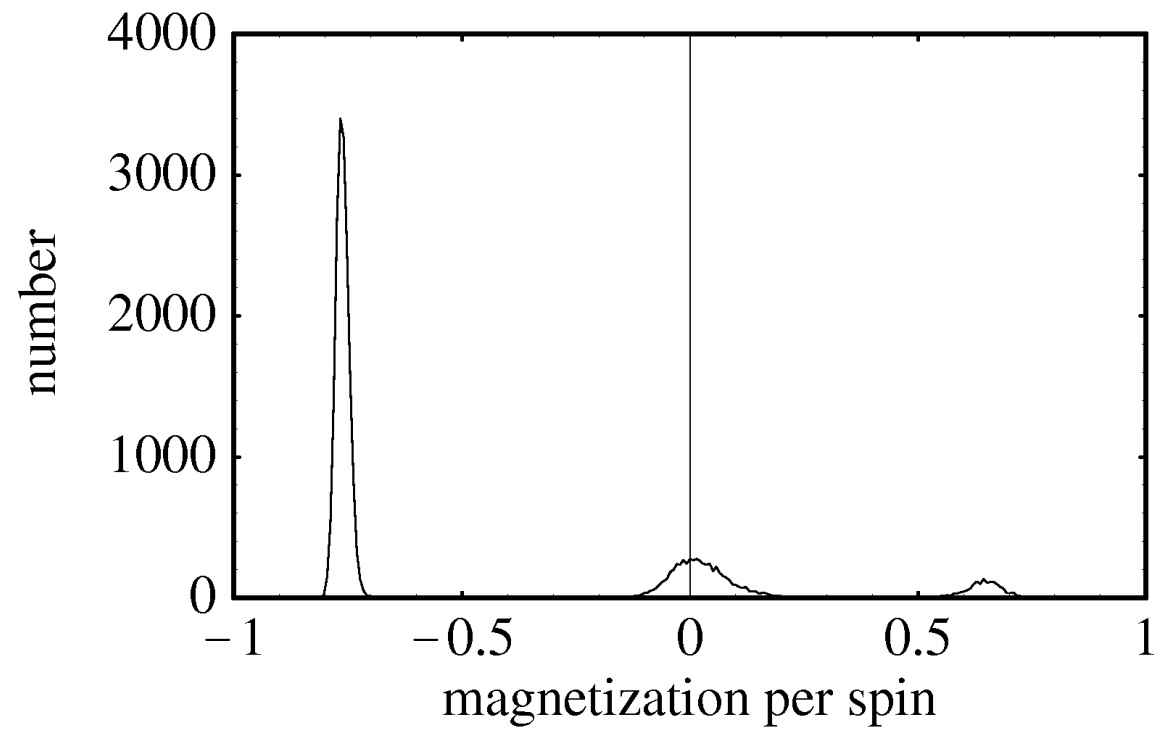

FIG. 6. Magnetization histogram for realization 31 at $\Delta=0.3267$. 


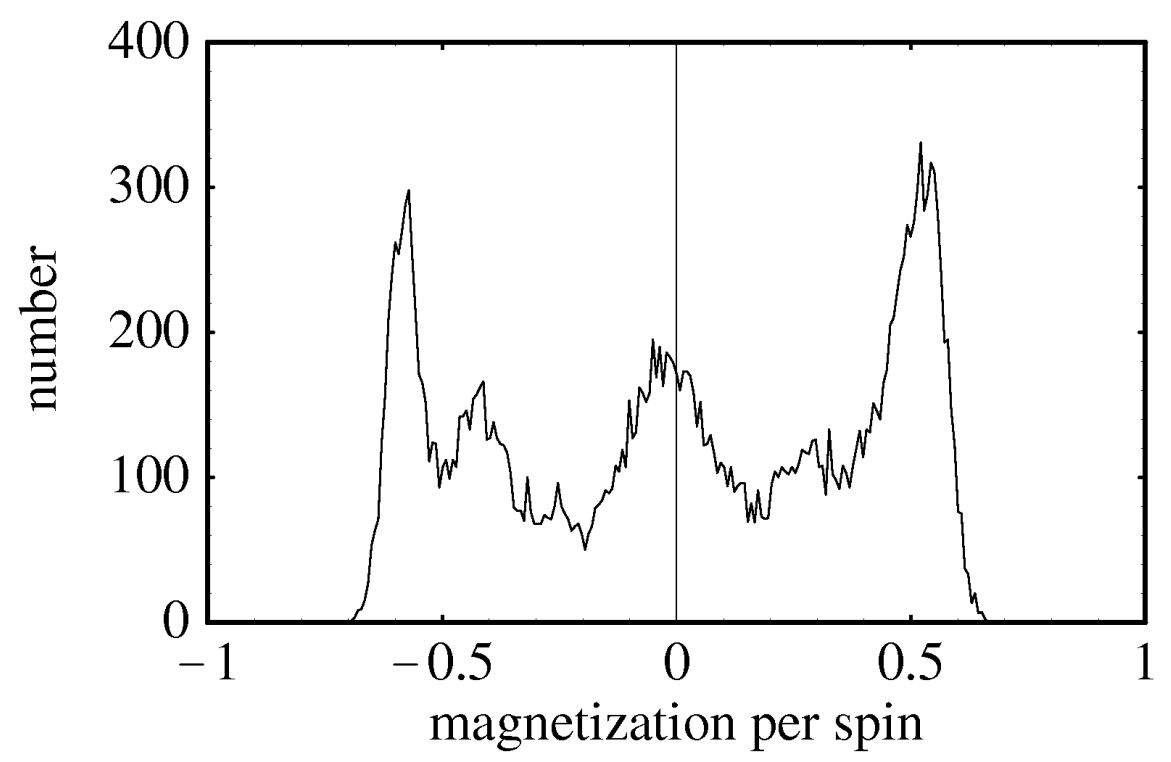

FIG. 7. Magnetization histogram for realization 14 and $\Delta=0.35$.

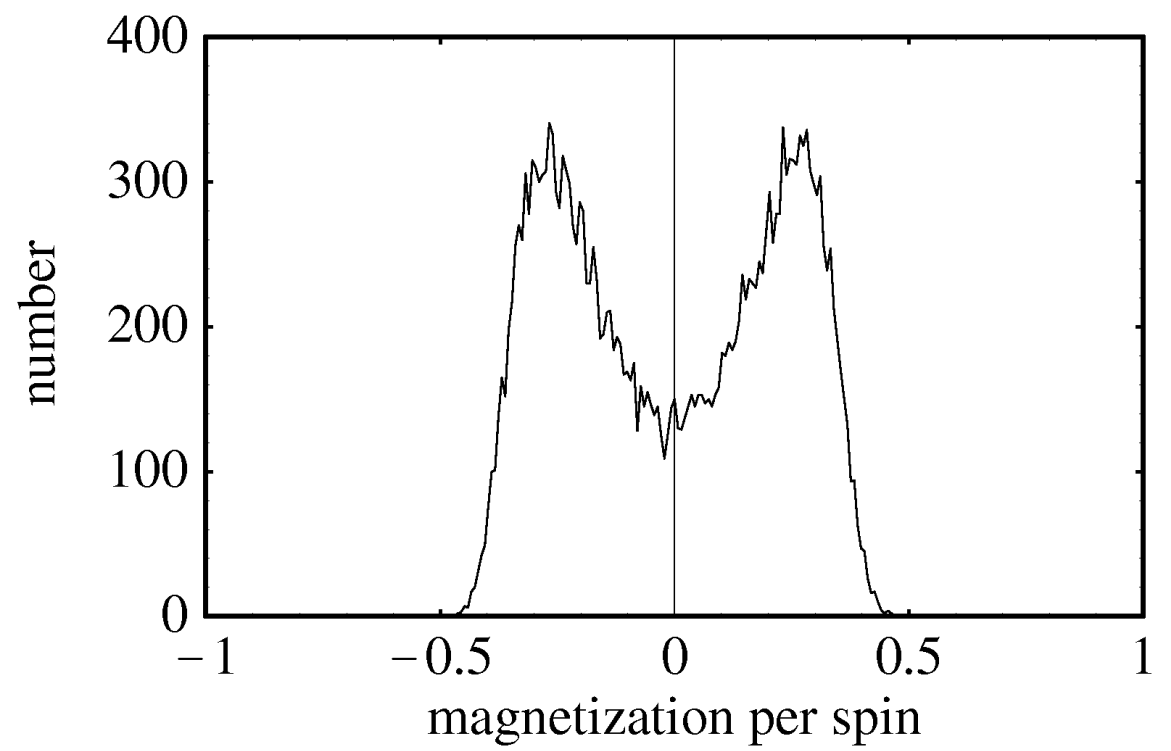

FIG. 8. Magnetization histogram for the pure Ising model at the infinite system size critical temperature $\beta_{c}=0.22165$. 


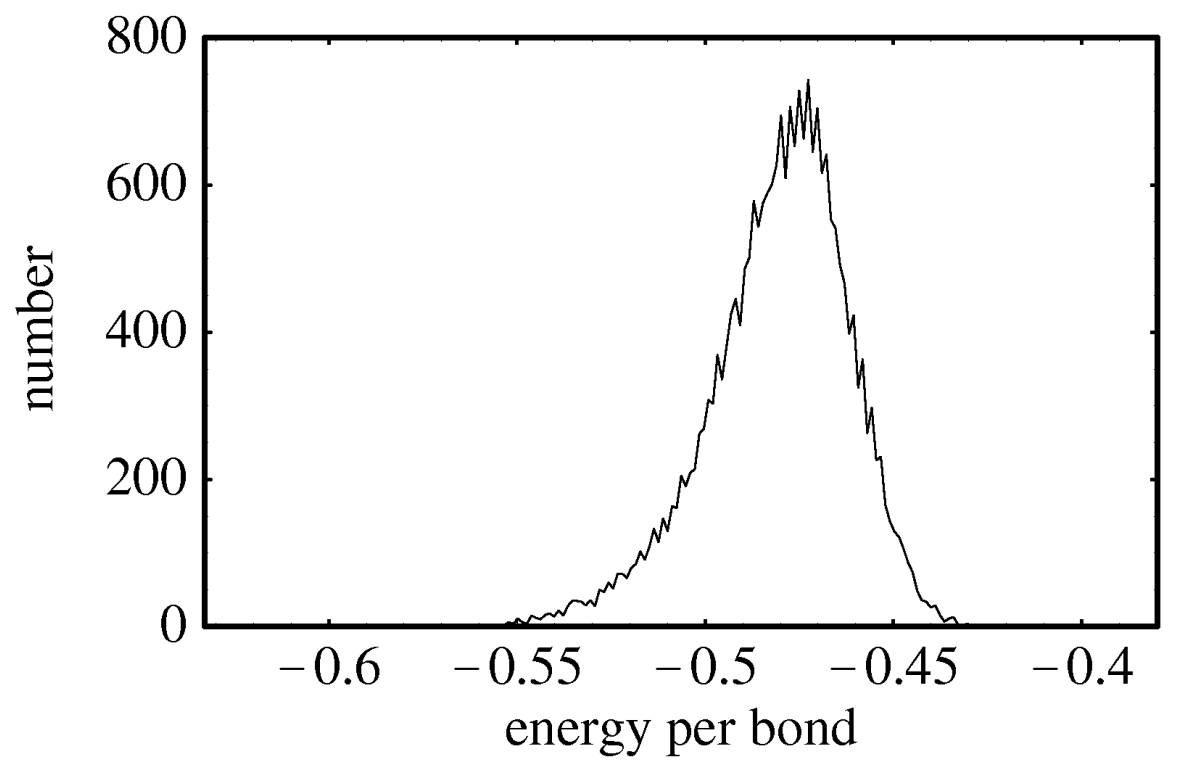

FIG. 9. Bond energy histogram for realization 20 at $\Delta=0.35$.

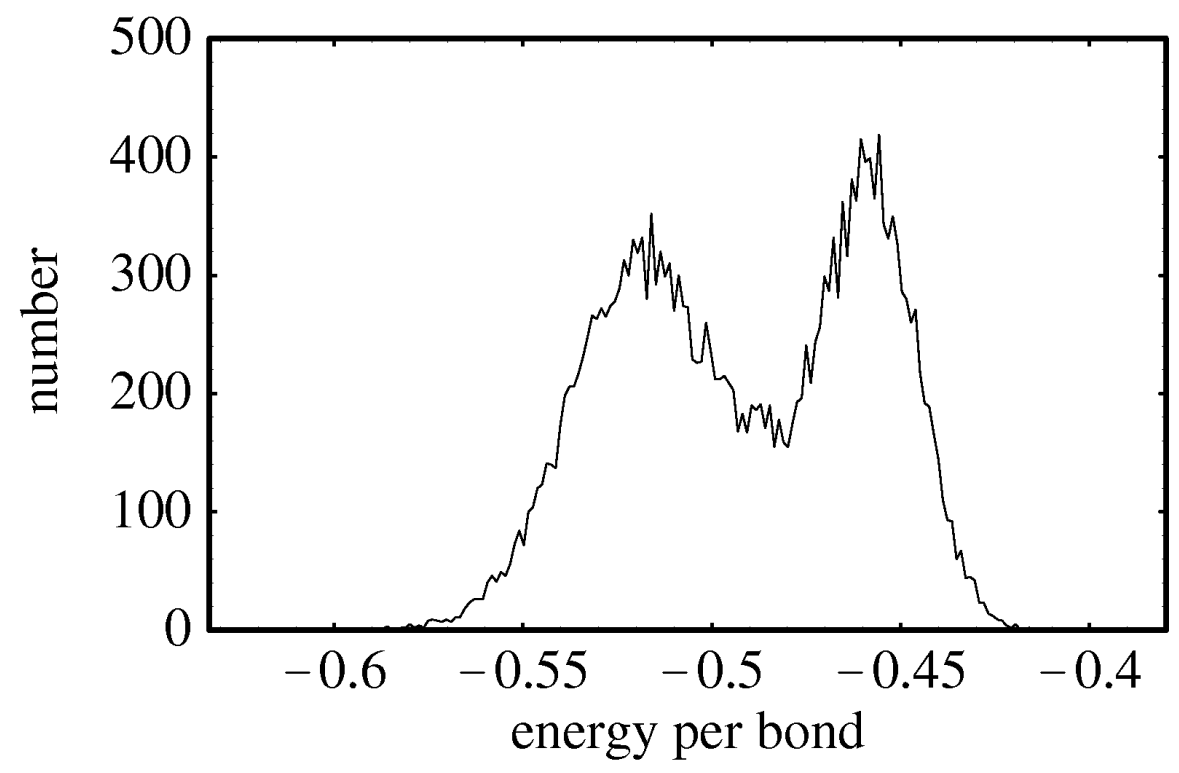

FIG. 10. Bond energy histogram for realization 21 at $\Delta=0.35$. 


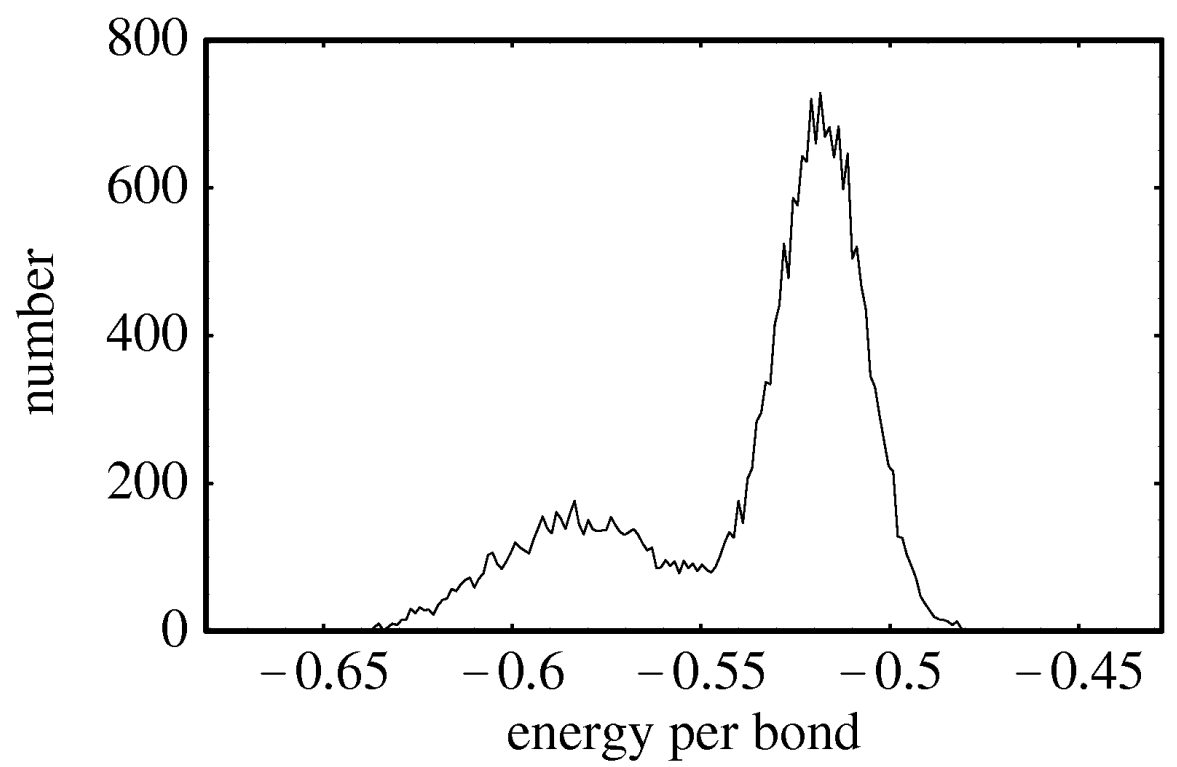

FIG. 11. Bond energy histogram for realization 25 at $\Delta=0.35$.

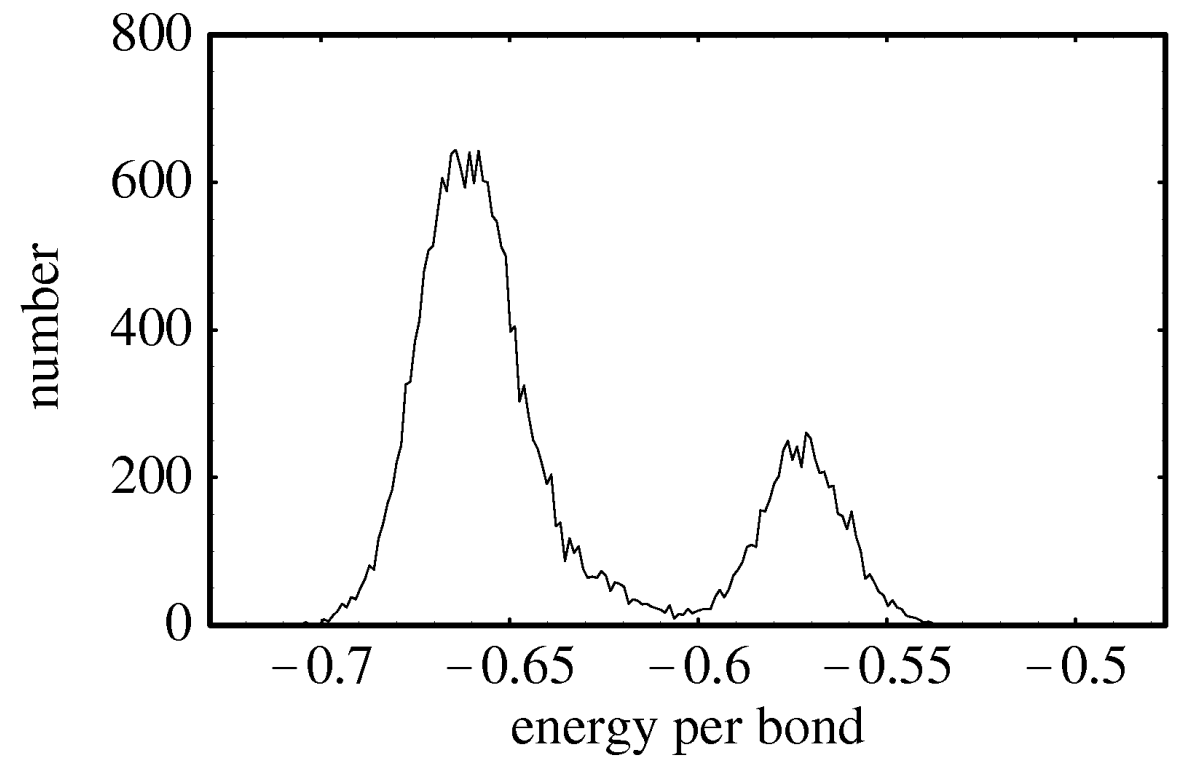

FIG. 12. Bond energy histogram for realization 31 at $\Delta=0.3267$. 


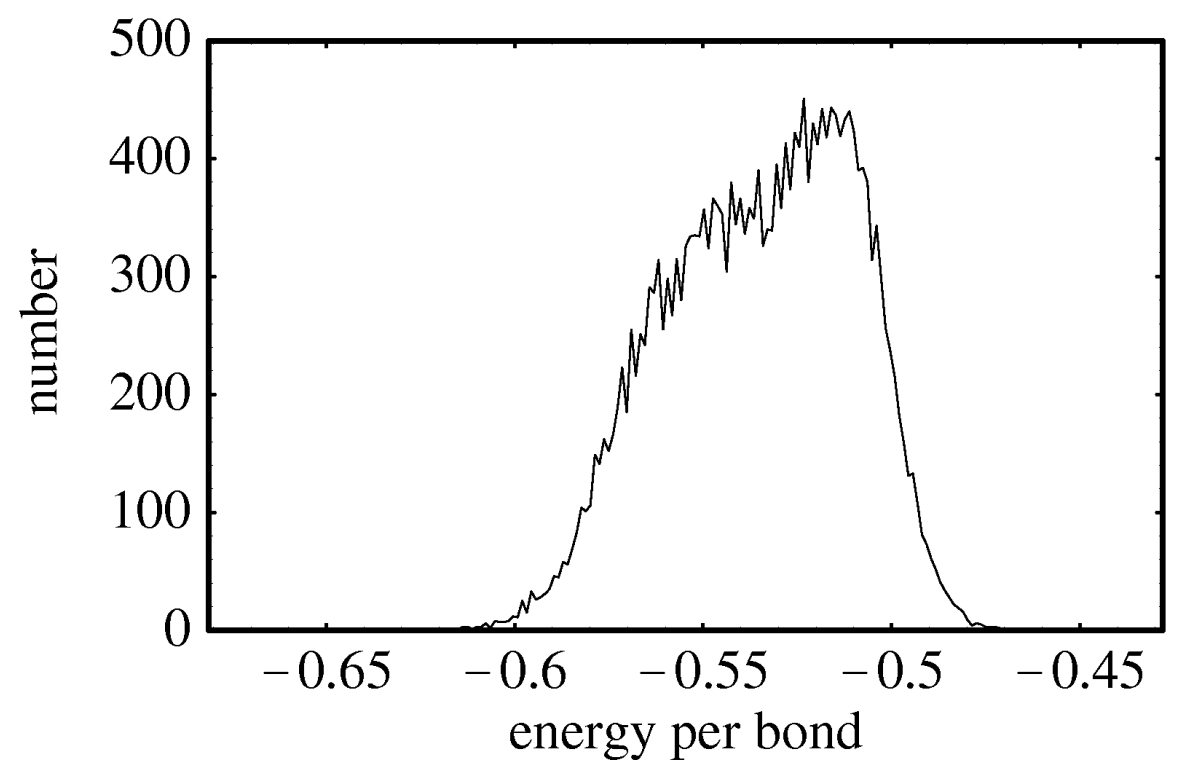

FIG. 13. Bond energy histogram for realization 14 at $\Delta=0.35$.

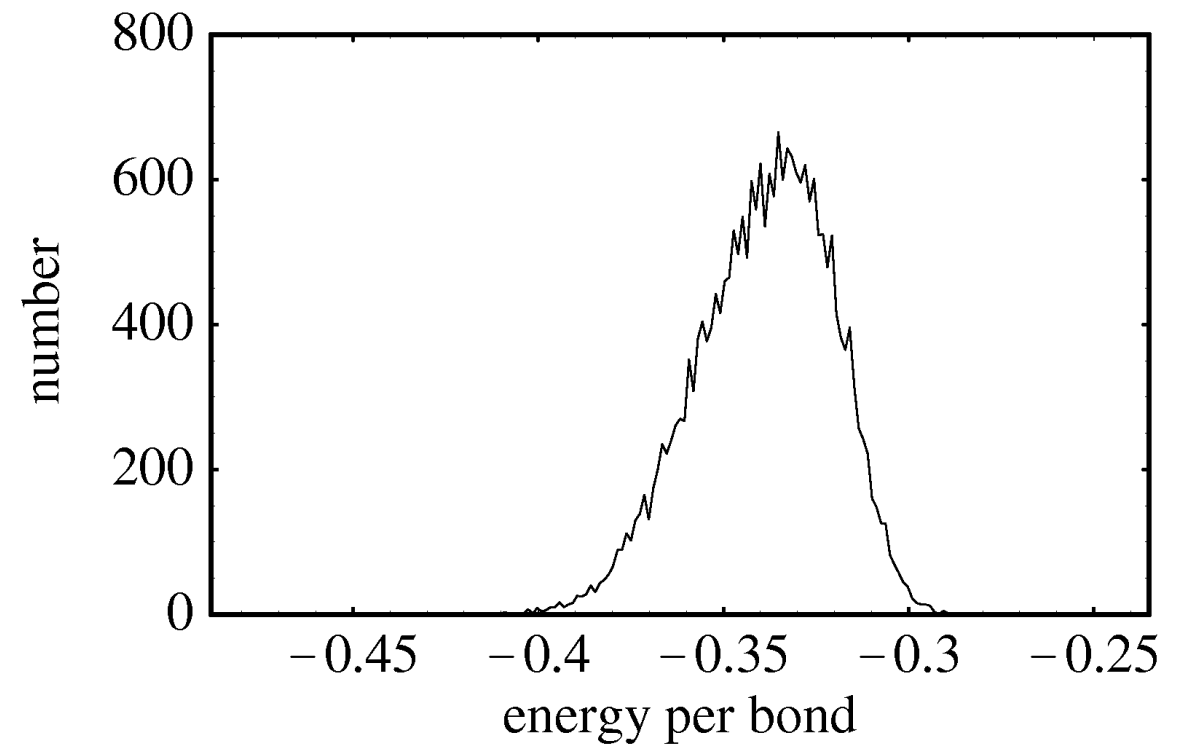

FIG. 14. Bond energy histogram for the pure Ising model at the infinite system size critical temperature $\beta_{c}=0.22165$. 


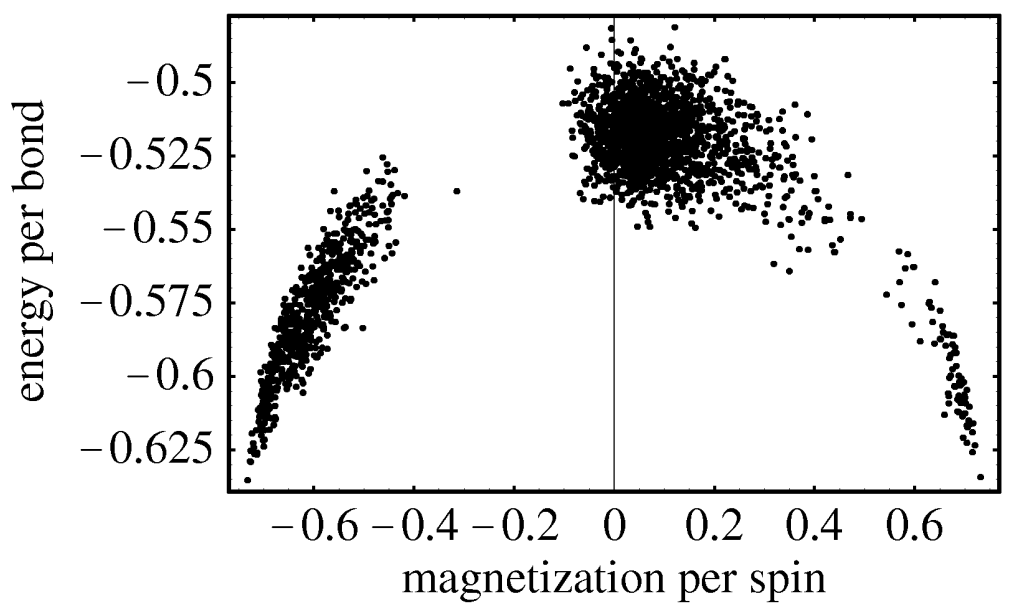

FIG. 15. The joint magnetization/bond-energy distribution for realization 25 at $\Delta=0.35$.

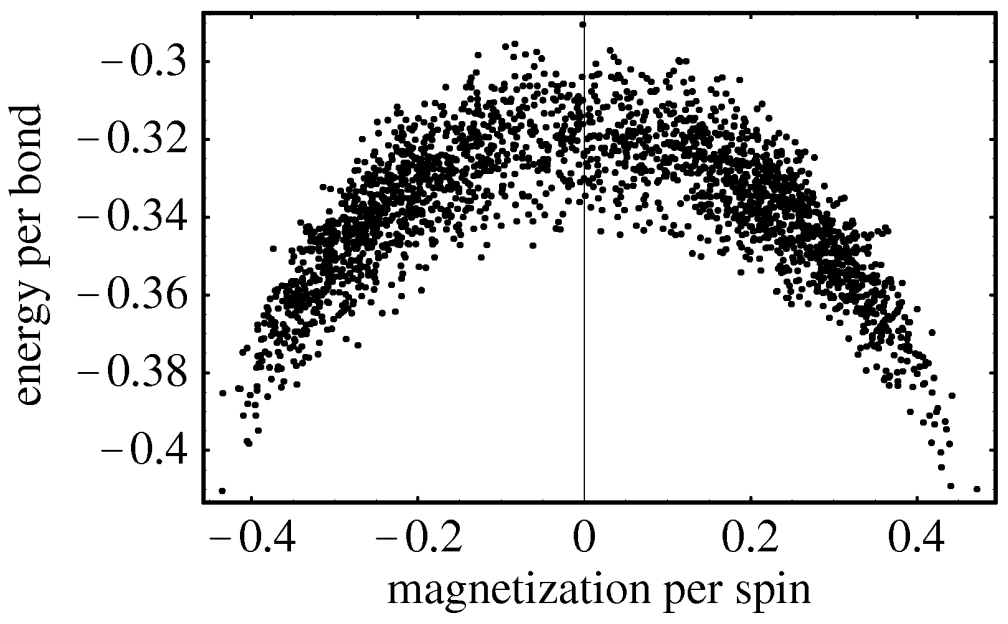

FIG. 16. The joint magnetization/bond-energy distribution for for the pure Ising model at the infinite system size critical temperature $\beta_{c}=0.22165$. 


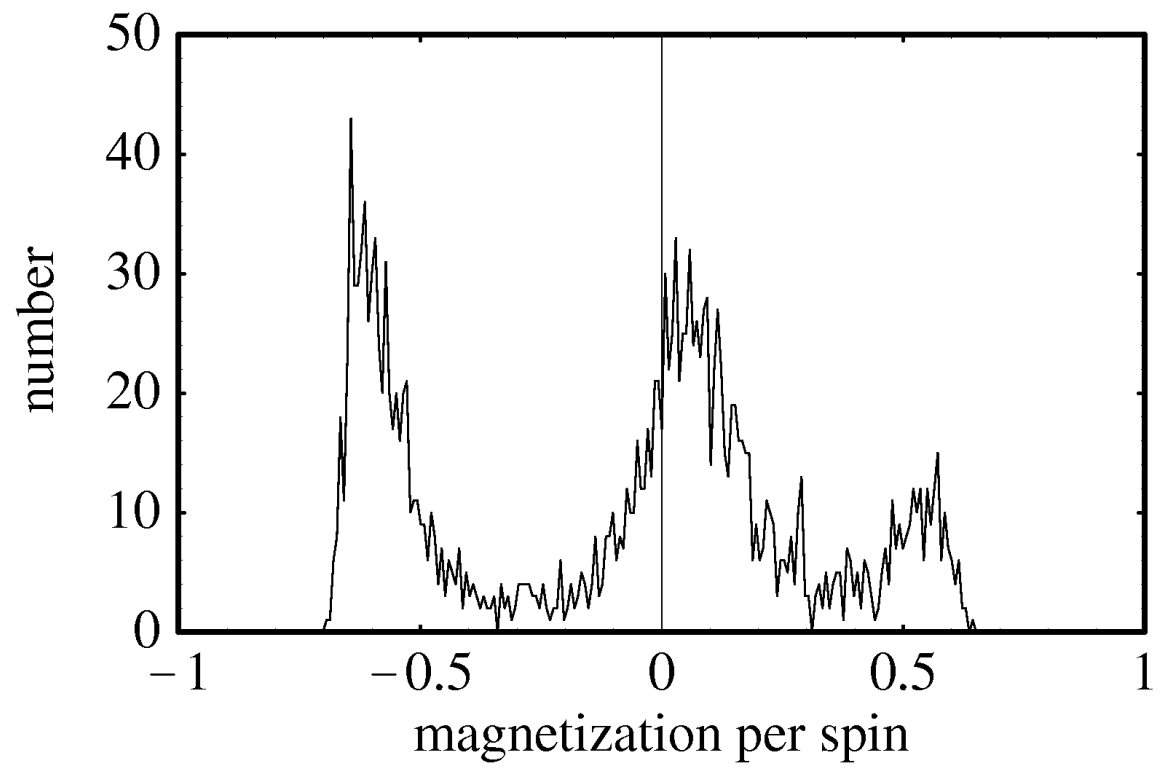

FIG. 17. Magnetization histogram for realization 1 at $\Delta=0.35$ and the phase transition point determined from fine-tuning, $\beta_{c}=0.268385$ and $H_{c}=0.00127049$.

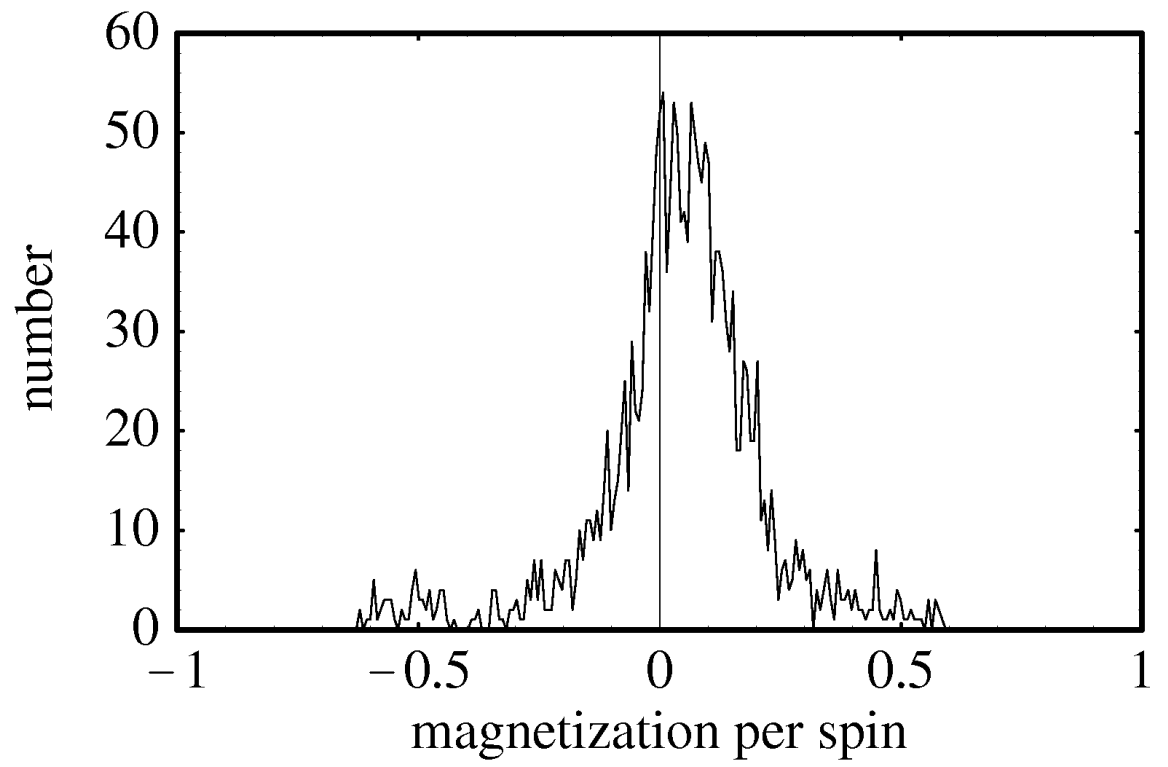

FIG. 18. Magnetization histogram for realization 1 at $\Delta=0.35$ and $\beta=0.267041$ (5\% warmer than $\beta_{c}$ determined for realization 1) and $H_{c}=0.00127049$. 


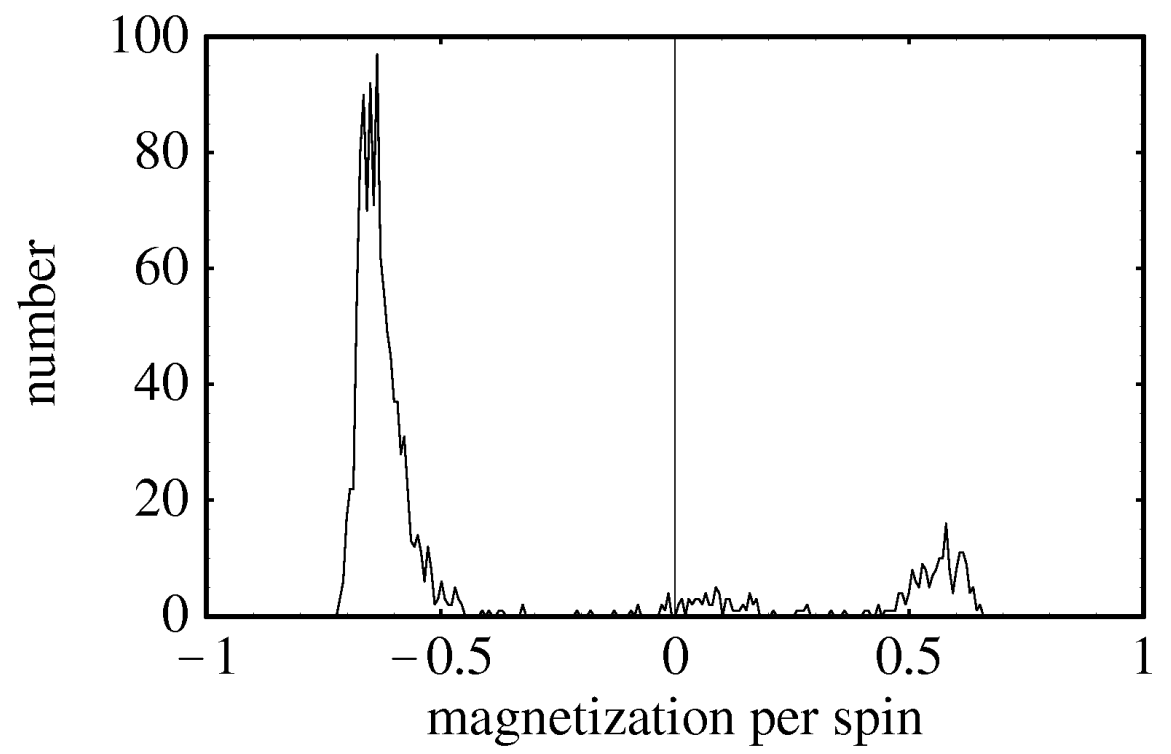

FIG. 19. Magnetization histogram for realization 1 at $\Delta=0.35$ and $\beta=0.269727$ (5\% colder than $\beta_{c}$ determined for realization 1) and $H_{c}=0.00127049$.

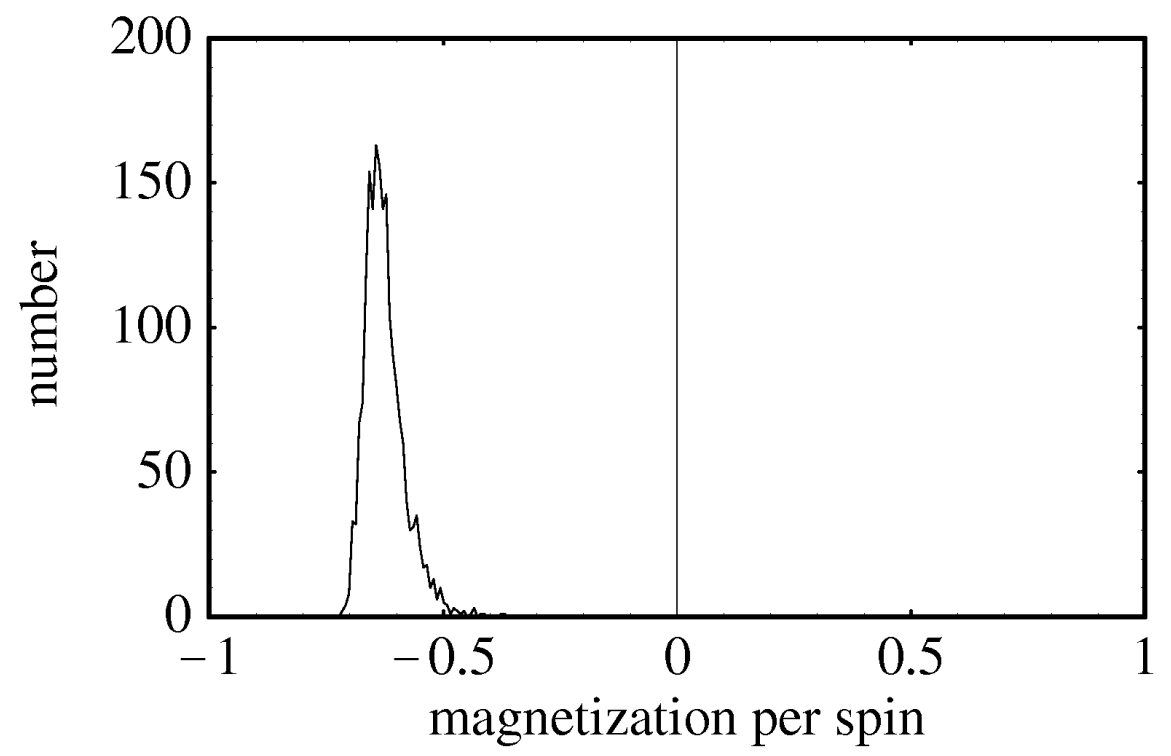

FIG. 20. Magnetization histogram for realization 1 at $\Delta=0.35, \beta_{c}=0.267041$ and $H=0$. 


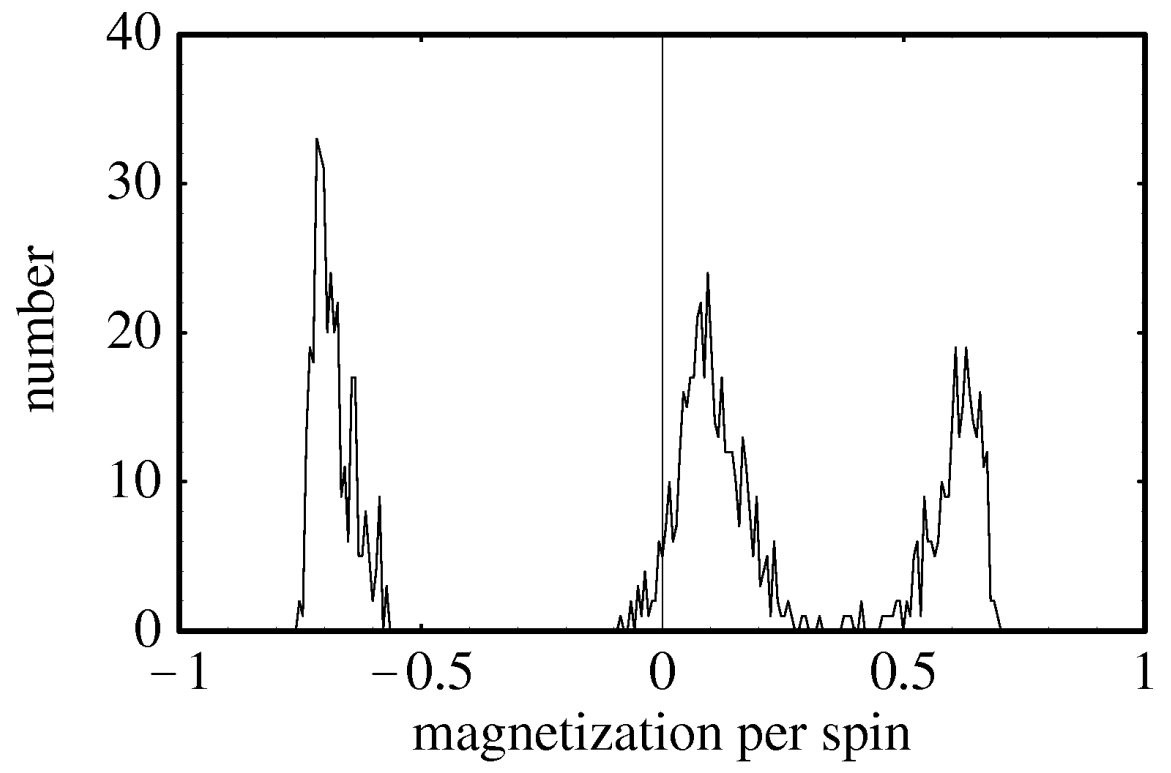

FIG. 21. Magnetization histogram for realization 1 at $\Delta=0.433$ at temperature and external field determined from fine-tuning, $\beta_{c}=0.289176$ and $H_{c}=0.00158005$.

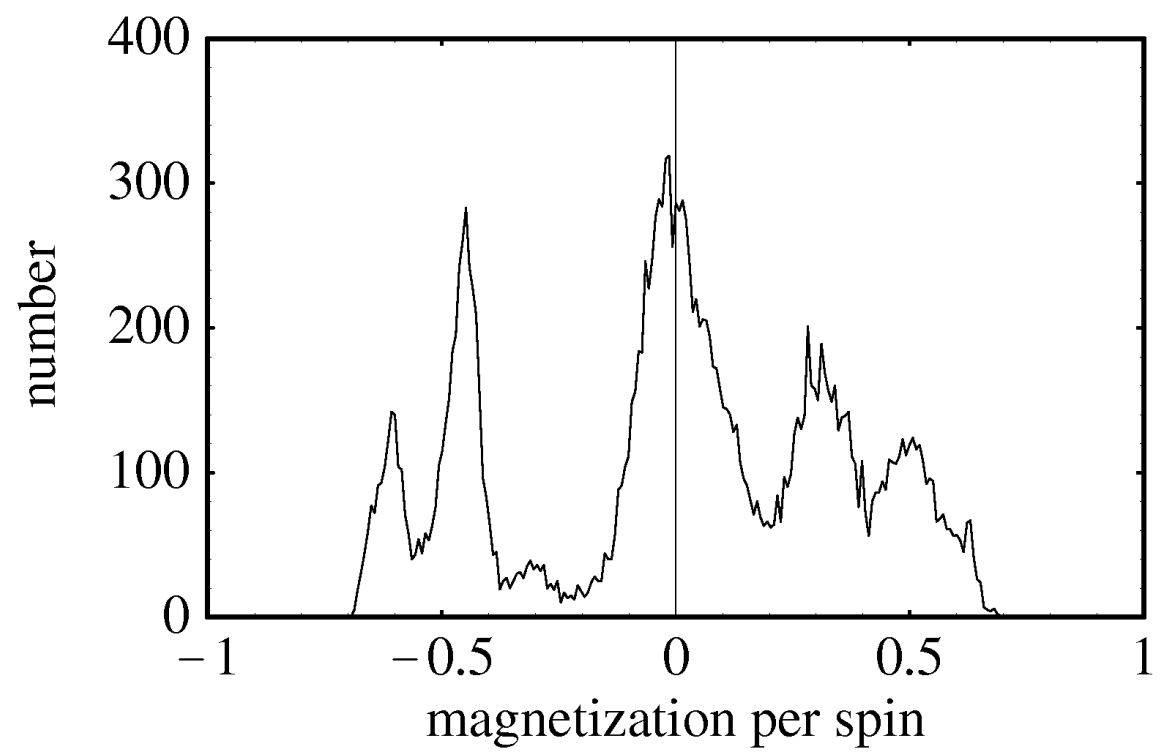

FIG. 22. Magnetization histogram for realization 14 at $\Delta=0.5$ at temperature and external field determined from fine-tuning. 


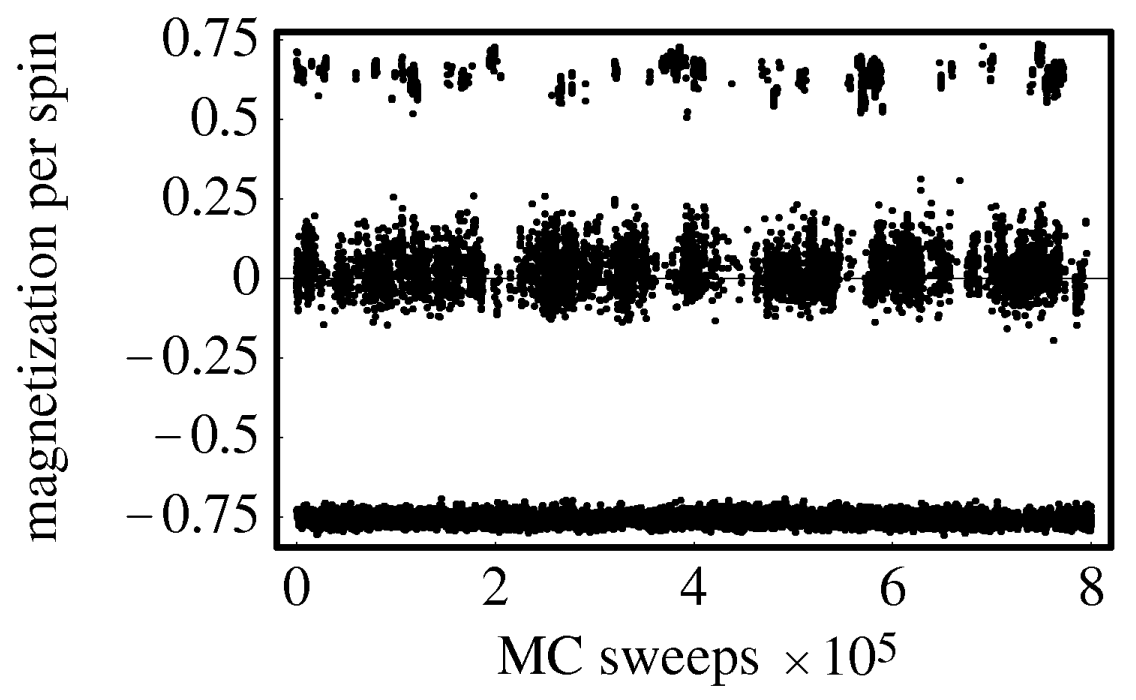

FIG. 23. Magnetization time series for realization 31 for $\Delta=.3267$. 